\title{
Una nuova metodica di rimozione del biofilm con polvere di eritritolo guidata da rilevatore di placca
}

\section{A new method for biofilm removal with erythritol powder guided by disclosing agent}

M. Mensia, ${ }^{*}$, M. Dalè ${ }^{b}, A$. Sordillo ${ }^{b}$

a Università degli Studi di Brescia, Dipartimento di Specialità Chirurgiche, Scienze Radiologiche e Sanità Pubblica, Divisione di Odontoiatria

b Università degli Studi di Brescia

Ricevuto il

28 novembre 2016

Accettato il

17 gennaio 2017

*Autore di riferimento Magda Mensi

magda.mensi@unibs.it
RIASSUNTO

OBIETTIVI. II presente lavoro valuta l'efficacia della rimozione del biofilm mediante polvere di eritritolo (Erythritol Powder Air Polishing Therapy, EPAPT) rispetto alla rimozione meccanica professionale (Professional Mechanical Plaque Removal, PMPR) con ultrasuoni, coppette e paste abrasive.

MATERIALI E METODI. Un soggetto sano, durante una seduta di profilassi, è stato trattato in split-mouth per confrontare le due metodiche guidate dal rilevatore di placca. Foto pre e post-trattamento e un questionario di feedback sono stati raccolti e valutati.

RISULTATI E CONCLUSIONI. La metodica EPAPT è risultata migliore in termini di percezione da parte del paziente, fastidio al trattamento, tempo di esecuzione ed efficacia nella rimozione della placca. II trattamento è invece risultato sovrapponibile in termini di sensazione di pulito.

\section{PAROLE CHIAVE}

- Eritritolo

- Rimozione della placca

- Rilevatore di placca

- Igiene orale

- Erythritol Powder Air Polishing Therapy (EPAPT)

\section{ABSTRACT}

OBJECTIVES. The aim of this study was to assess the biofilm removal efficacy of Erythritol Powder Air Polishing Therapy (EPAPT) compared to Professional Mechanical Plaque Removal (PMPR) with ultrasonic device, rubber cup and abrasive paste.

MATERIALS AND METHODS. A healthy patient received a session of prophylaxis using a split-mouth design in order to compare these two methods guided by a disclosing agent. Pre and post-treatment photos and a feedback questionnaire were collected and evaluated. 
RESULTS AND CONCLUSIONS. EPAPT proved to be more effective than PMRP in terms of comfort, pain felt during treatment, time of execution and efficacy in plaque removal.

EPAPT and PMPR proved to be compa- rable in terms of cleanliness feeling perceived by the patient.

\section{KEY WORDS}

- Erythritol

- Plaque removal
- Disclosing agent

- Oral hygiene

- Erythritol Powder Air Polishing

Therapy (EPAPT)

\section{INTRODUZIONE}

La prevenzione primaria e secondaria, insieme alla terapia parodontale attiva, rappresenta il fondamento della parodontologia. Parte importantissima delle sedute di profilassi e terapia di supporto è la fase motivazionale e di istruzione all'igiene orale domiciliare, che risulta strettamente correlata al successo e al mantenimento a lungo termine della salute parodontale. Trovare metodi di trattamento confortevoli, veloci, efficaci e sicuri di rimozione del biofilm e dell'eventuale tartaro presente è importante, perciò, per fidelizzare il paziente stimolandolo a tornare ai richiami senza paura di sentire dolore e per garantire all'igienista dentale il tempo necessario alla raccolta dei dati clinici necessari alla valutazione del rischio, all'identificazione di eventuali problematiche di nuova insorgenza e per la motivazione e istruzione.

La rimozione meccanica della placca (Professional Mechanical Plaque Removal, PMPR) [1] permette di disgregare i depositi sia sopra sia sottogengivali con conseguente stabilizzazione dell'equilibrio tra la popolazione batterica e la risposta dell'ospite [2]. La metodica attuale si basa sull'utilizzo di strumenti meccanici e/o manuali per la rimozione di placca e tartaro [3] e la lucidatura con coppette e paste abrasive. L'impiego di questi strumenti può causare l'asporta- zione di tessuti duri [4] quali il cemento e la dentina. Ciò determina alcune problematiche quali l'aumento di rugosità della superficie trattata [5], recessioni, ipersensibilità [6] e fastidio durante il trattamento [7]. La presenza di questi effetti negativi ha portato alla ricerca di un diverso approccio all'eliminazione del biofilm.

Il bicarbonato di sodio ad alta granulometria (40-105 $\mu \mathrm{m})$ permette la rimozione della placca e delle macchie sopragengivali ma risulta molto aggressivo nei confronti dei tessuti molli e duri [8]. Sono state successivamente introdotte polveri a bassa abrasività, quali la glicina e in seguito l'eritritolo (25 e $14 \mu \mathrm{m}$, rispettivamente), utilizzabili per il trattamento sia sopragengivale sia sottogengivale [9]. L’impiego di tali polveri ha comportato diversi vantaggi tra cui la diminuzione del fastidio e discomfort provati dal paziente, il minor tempo di esecuzione del trattamento, la possibilità di pulire zone di difficile accesso e soprattutto un minor danno a livello dei tessuti molli e duri.

Ł̀ stato inoltre evidenziato che l'eritritolo possiede una struttura interferente con la formazione della microstruttura del biofilm di Streptococcus gordonii e Porphyromonas gingivalis [10].

L'obiettivo dello studio è quello di comparare l'efficacia del nuovo protocollo (Erythritol Powder Air Polishing Therapy, EPAPT) che prevede la rimozione del biofilm sopra e sottogengivale tramite polvere di eritritolo rispetto alla metodica tradizionale (PMPR)

\section{MATERIALI E METODI}

È stata selezionata una paziente sistemicamente e parodontalmente sana, non fumatrice, periodicamente seguita per sedute di profilassi.

Dopo aver effettuato il sondaggio parodontale per escludere la presenza di tasche, pseudotasche e sanguinamento marginale diffuso e dopo aver fatto eseguire uno sciacquo con clorexidina allo 0,12\% per ridurre la carica microbica nell'aerosol indotto dal trattamento, si è posizionato un retrattore tipo OptraGate $^{\circledR}$ (Ivoclar Vivadent Manufacturing Srl, Naturno, Bolzano) per proteggere le labbra, scostare i tessuti molli e poter applicare il rilevatore di placca senza interferenze.

La terapia è stata svolta in split-mouth: i quadranti 2 e 3 con metodica PMPR, i quadranti 1 e 4 con metodica EPAPT.

Sono state eseguite fotografie cliniche (frontali, linguali, palatali della sestina anteriore superiore e inferiore) pretrattamento (fig. 1a-c) e dopo il posizionamento del rilevatore di placca (Mira-2-Ton ${ }^{\circledR}$, liquido rivelatore, flacone da $60 \mathrm{~mL}$, Hager \& Werken GmbH, Duisburg, DE) per valutare l'indice di placca iniziale (fig. 2a-c). Nei quadranti in cui è stata praticata la PMPR (settori 2 e 3) è stato inizialmente 

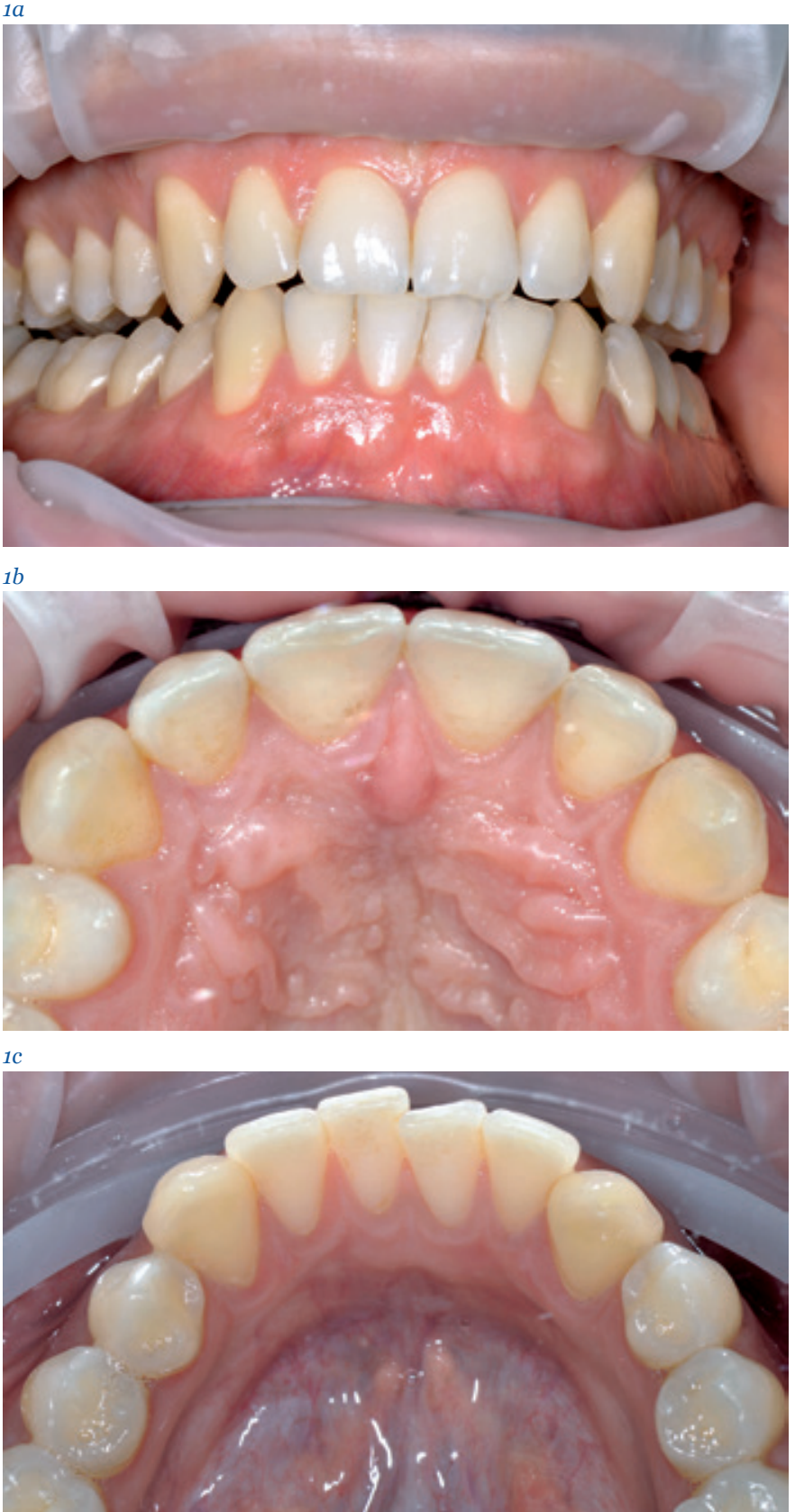

Fig. 1a-c Foto pretrattamento. a) Visione frontale: si noti la recessione sul 2.3 che rappresenta un punto di difficoltà all'uso dell'ablatore. b) Visione palatale. c) Visione linguale
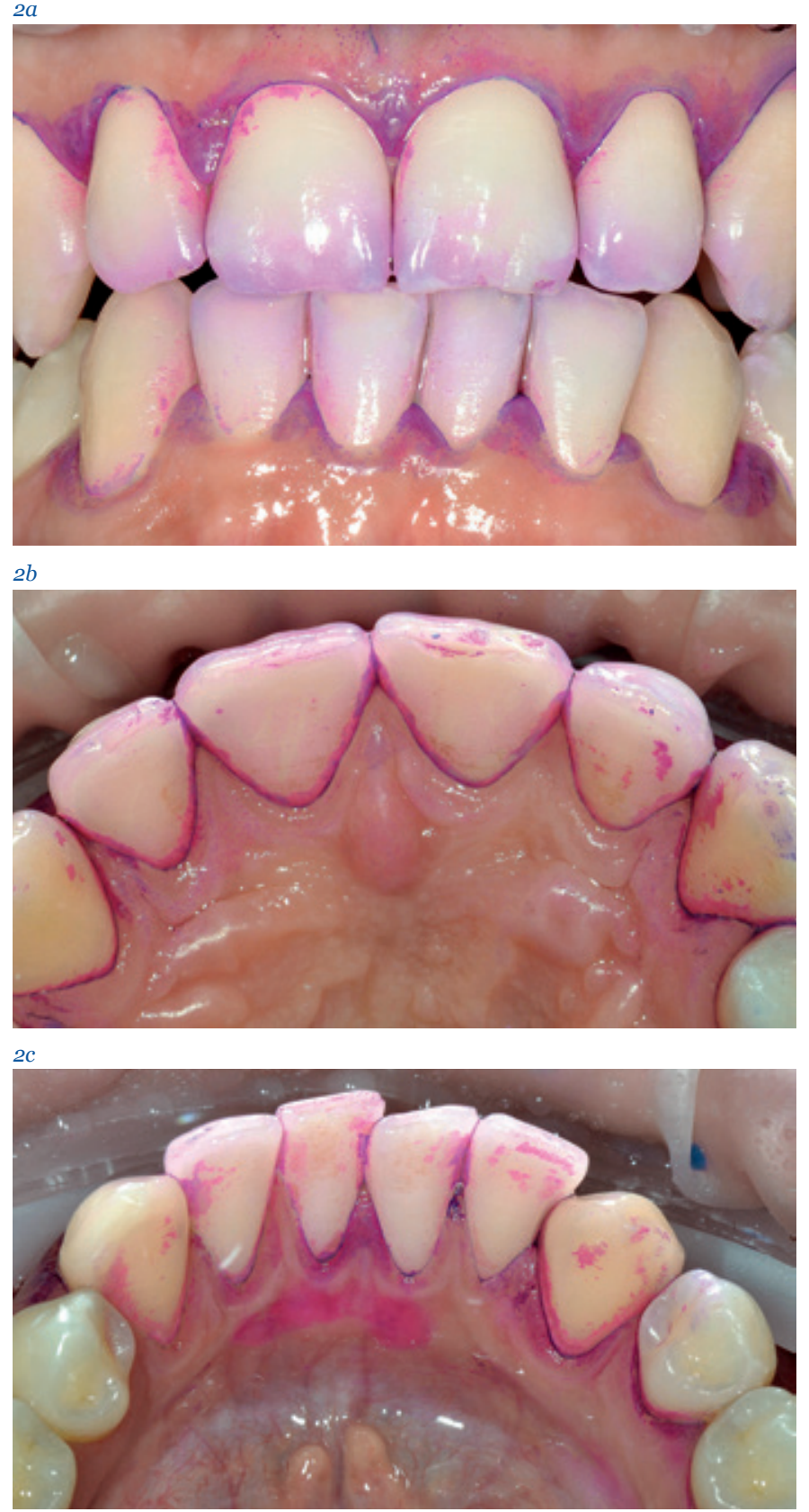

Fig. 2a-c Foto pretrattamento con rilevatore di placca.

a) Visione frontale: si noti il rilevatore di placca che si colora in maniera differente a seconda del suo livello di maturazione. b) Visione palatale. c) Visione linguale utilizzato un manipolo piezoelettrico con punta Perio Slim (PS ${ }^{\circledR}$, EMS SA, Nyon, $\mathrm{CH}$ ). Le superfici dentali sono state lucidate con coppette soft (coppetta palmata Krugg, attacco CA) e pasta abrasiva a bassa granulometria (Nupro ${ }^{\circledR}$ grana fine, Dentsply).

Nei quadranti in cui è stata eseguita la EPAPT (settori 1 e 4) sono stati effettuati i seguenti passaggi:
- decontaminazione della gengiva aderente tramite polvere di eritritolo (Plus $^{\circledR}$, EMS SA) con manipolo sopragengivale, inclinazione apico-coronale di $30^{\circ}$, a pressione minima $(35 \% \mathrm{su}$ 
Air Flow Master Piezon ${ }^{\circledR}$, EMS SA) e movimenti rapidi di vai e vieni. Irrigazione massima;

- decontaminazione sopragengivale per rimuovere biofilm e macchie con polvere di eritritolo (Plus ${ }^{\circledR}$, EMS SA) con manipolo sopragengivale, inclinazione apico-coronale di $45^{\circ}$, pressione medio-massima in base alla tenacità delle macchie e della placca (50-100\% su Air Flow Master Piezon $^{\circledR}$, EMS SA). Irrigazione massima;

- decontaminazione sottogengivale nei solchi con polvere di eritritolo (Plus ${ }^{\circledR}$, EMS SA) a bassa pressione (35-50\% su Air Flow Master Piezon ${ }^{\circledR}$, EMS SA) in senso corono-apicale verso il solco, con movimenti di vai e vieni, a $5 \mathrm{~mm}$ di distanza e per circa 5 secondi per sito. Irrigazione massima.

I trattamenti sono stati eseguiti fino al raggiungimento di un risultato soddisfacente per l'operatore, corrispondente alla completa rimozione del rivelatore di placca. È stato inoltre annotato il tempo di esecuzione dei due differenti trattamenti. Sono state eseguite ulteriori fotografie post-trattamento (fig. 3a-c). A questo punto è stato applicato nuovamente il rilevatore di placca e sono state scattate le fotografie post-trattamento finali per la valutazione dell'indice di placca residua (fig. 4a-c).

Alla paziente è stato somministrato un questionario per valutare la sensazione di pulito, il fastidio provato e il comfort percepito nei due trattamenti. Per la valutazione del dolore è stata utilizzata una scala da 1 a 5: 1 equivaleva all'assenza di dolore, 5 alla sensazione di dolore massimo. Le altre variabili sono state quantificate con una scala da 1 a 5 , da insufficiente a ottimo. Alla fine del questionario si è chiesto alla paziente di scegliere il trattamento che avrebbe preferito ricevere alla
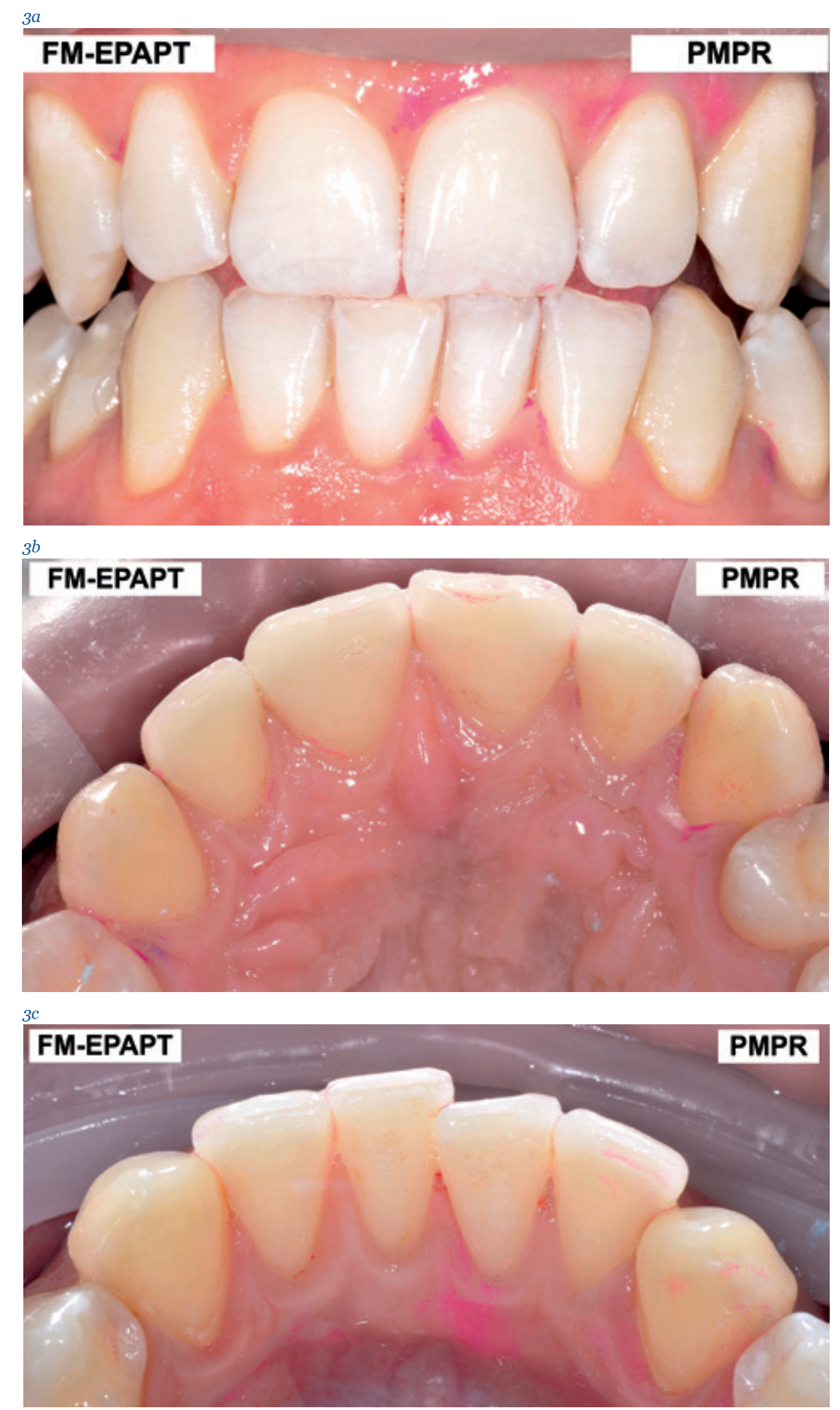

Fig. 3a-c Foto post-trattamento. a) Visione frontale, apparentemente sono stati rimossi completamente la placca e il tartaro. b) Visione palatale. c) Visione linguale 


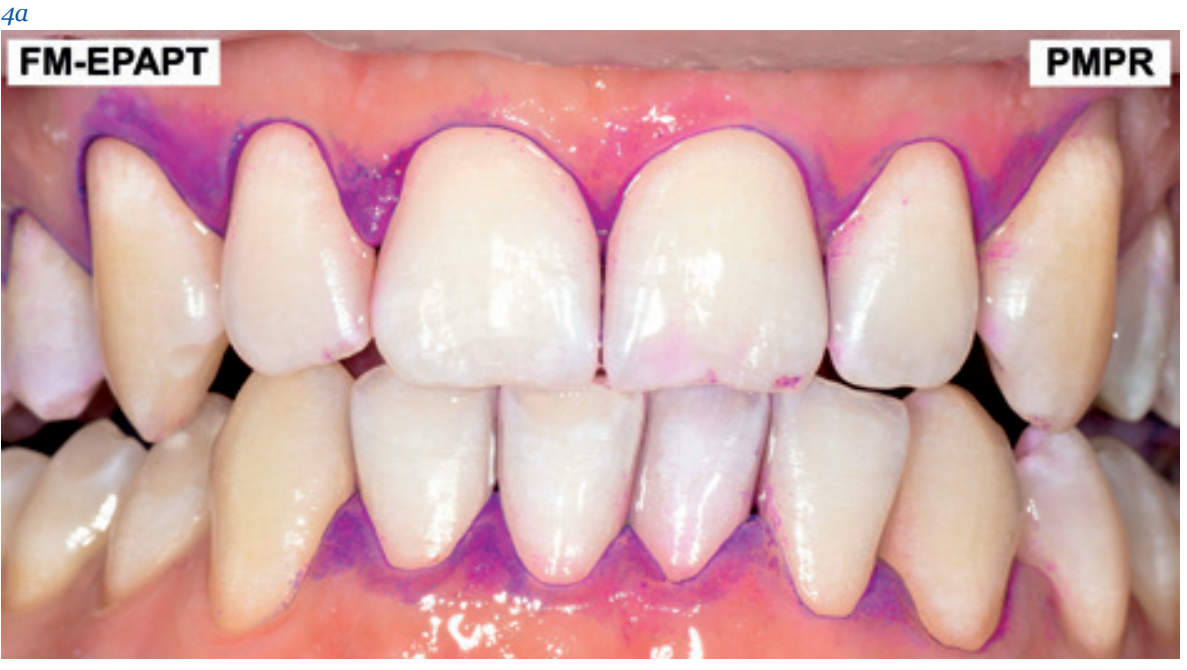

$4 b$

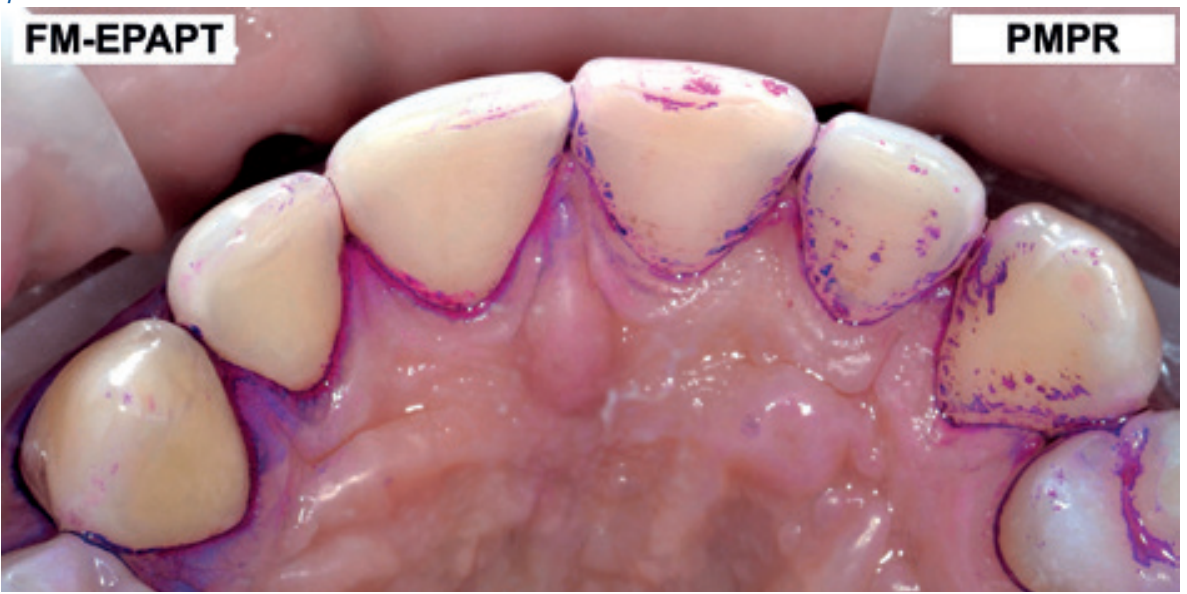

$4 c$

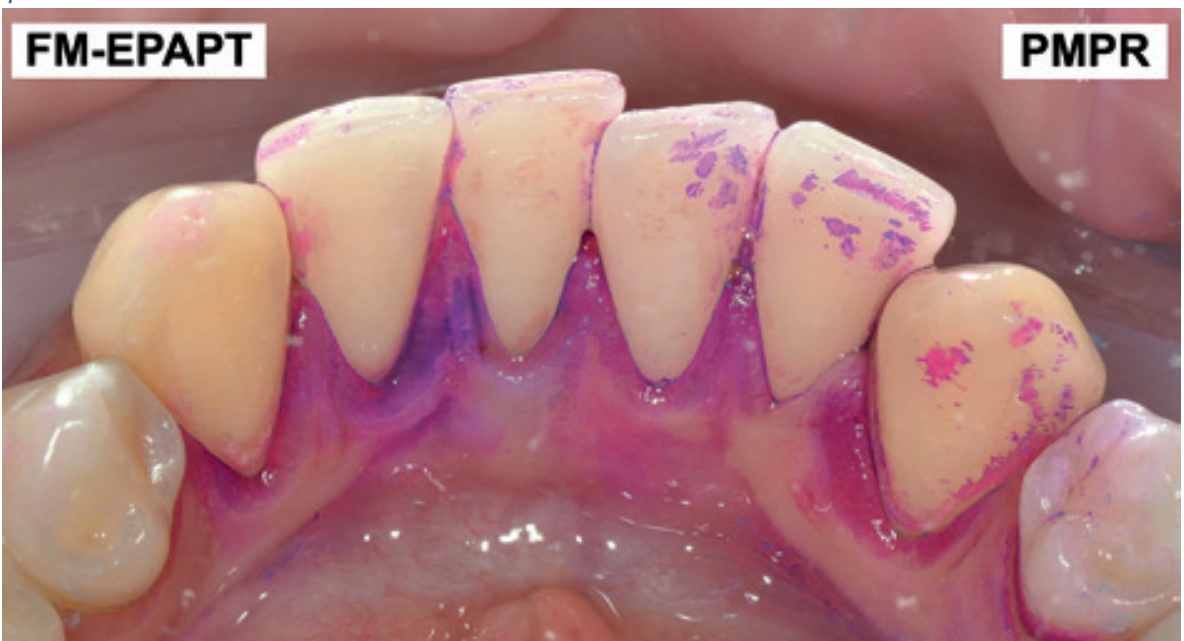

Fig. 4a-c Si noti la differenza dei residui a seconda del trattamento. a) Foto post-trattamento frontale con rilevatore di placca: le porzioni colorate rappresentano aree in cui il trattamento non è stato efficace. b) Foto post-trattamento palatale con rilevatore di placca. c) Foto post-trattamento linguale con rilevatore di placca seduta successiva. La stessa scala di valutazione è stata utilizzata per quantificare il comfort dell'operatore nell'esecuzione del trattamento.

\section{RISULTATI}

Le due metodiche sono risultate sovrapponibili per quanto riguarda la sensazione di pulito $(\mathrm{PMPR}=5 ; \mathrm{EPAPT}=5)$.

$\mathrm{Si}$ è riscontrata una differenza rilevante riguardo al fastidio percepito dalla paziente: nella PMPR il valore è stato pari 2, mentre nella EPAPT pari a o. Ciò dimostra il maggior comfort della paziente durante il trattamento con eritritolo.

Analizzando il tempo impiegato, è stato di 12' 26" per la PMPR e 9' 34 " per la EPAPT.

Il comfort dell'operatore è stato quantificato 4 per il trattamento PMPR e 5 per la metodica EPAPT, dimostrando una lieve preferenza per la nuova metodica.

L'efficacia in termini di rimozione di placca, valutata riapplicando il rilevatore di placca dopo le terapie, è risultata superiore nei settori trattati con EPAPT.

\section{DISCUSSIONE}

I trattamenti di profilassi e supporto devono essere eseguiti, a cadenza costante, per tutta la vita del paziente e con frequenza personalizzata sulla base del profilo di rischio individuale. Da ciò si può evincere la necessità che le metodiche di igiene professionale risultino il più possibile confortevoli, veloci e meno fastidiose. È inoltre preferibile applicare metodiche che determinino il minor danno possibile ai tessuti duri e molli del cavo orale.

L'uso del rilevatore di placca svolge un ruolo fondamentale al fine di ottenere una completa rimozione del biofilm e 
della placca batterica in maniera selettiva e mininvasiva, guidando l'operatore nel tempo e nel modo di utilizzo dei dispositivi.

Si può notare come alcune superfici dentali siano difficili da raggiungere con le coppette soft, soprattutto su denti che presentano rotazioni e/o spazi interprossimali stretti, siti palatali e linguali, così come nei pazienti ortodontici o implantoprotesici. L'eritritolo permette di accedere meglio a queste zone, completando in maniera efficace la rimozione del biofilm. L'utilizzo del rilevatore di placca consente oltretutto al clinico di motivare e istruire il paziente all'igiene orale domiciliare, di controllare il lavoro eseguito ed eventualmente di rifinire i siti dove non si è stati completamente efficaci. L'uso dell'airflow all'inizio del trattamento permette di ridurre da subito la carica microbica di denti e gengive, rimuovere le macchie, creare un campo pulito dove è più facile identificare eventuale tartaro sopra e sottogengivale e indebolire il tartaro stesso che diventa più facilmente removibile con punte sottili su ultrasuono, che verranno utilizzate in modo selettivo solo dove siano presenti depositi mineralizzati.

Nel caso in questione non si è reso necessario il ricorso all'ultrasuono in quanto non erano presenti depositi mineralizzati non asportabili mediante l'impiego della polvere di eritritolo. La seduta di profilassi si è perciò conclusa con l'EPAPT. Tale metodica costituisce la parte iniziale della più ampia concezione di terapia guidata del biofilm (Guided Biofilm Therapy, GBT) che, in base alle necessità del singolo paziente, può essere seguita dall'uso delle punte ultrasoni- che nei siti sopra e sottogengivali in cui è presente tartaro ed, eventualmente, dalle minicurette after-five nei pazienti con parodontite severa con tasche $>10 \mathrm{~mm}$, dove è impossibile raggiungere il fondo del difetto in terapia attiva con gli inserti ultrasonici disponibili sul mercato.

La filosofia della GBT si basa su gradi crescenti di invasività in relazione alla gravità della situazione clinica del paziente, quindi soggetti sani in profilassi, come il caso presentato, possono essere sottoposti alla sola fase EPAPT per essere mantenuti con successo.

\section{CONCLUSIONI}

Nel caso descritto il protocollo EPAPT ha permesso di ottenere risultati clinici superiori al trattamento tradizionale. I vantaggi di tale metodica sono associati 
alla minore invasività che permette un minor danno ai tessuti duri e molli del cavo orale, al maggior comfort per l'operatore e per il paziente, alla velocità del trattamento e alla possibilità di raggiungere siti altrimenti non decontaminabili. Tutto questo risulta importante al fine di ottenere un maggior livello di compliance e quindi una maggiore efficienza nella salvaguardia della salute orale.

Trial clinici randomizzati controllati dovranno essere condotti per confermare tali conclusioni.

\section{CONFLITTO DI INTERESSI}

Gli autori dichiarano di non avere alcun conflitto di interessi.

\section{FINANZIAMENTI ALLO STUDIO}

Gli autori dichiarano di non aver ricevuto finanziamenti per il presente studio.

\section{CONSENSO INFORMATO}

Nel caso descritto, gli autori hanno ottenuto il consenso informato al trattamento.

\section{BIBLIOGRAFIA}

1. Tonetti MS, Eickholz P, Loos BG, Papapanou $P$, van der Velden $U$, Armitage $G$, et al. Principles in prevention of periodontal diseases. Consensus report of group 1 of the 11th European Workshop on Periodontology on effective prevention of periodontal and peri-implant diseases. J Clin Periodontol 2015;42(Suppl 16):S5-11.

2. Cobb CM. Clinical significance of non-surgi- cal periodontal therapy: an evidence-based perspective of scaling and root planing. J Clin Periodontol 2002;29(Suppl 2):6-16.

3. Worthington HV, Clarkson JE, Bryan G, Beirne PV. Routine scale and polish for periodontal health in adults. Cochrane Database Syst Rev 2013;(11):CD004625.

4. Flemmig TF, Petersilka GJ, Mehl A, Hickel $\mathbf{R}$, Klaiber $\mathbf{B}$. The effect of working parameters on root substance removal using a piezoelectric ultrasonic sealer in vitro. J Clin Periodontol 1998;25(2):158-63.

5. Kumar P, Das SJ, Sonowal ST, Chawla J. Comparison of root surface roughness produced by hand instruments and ultrasonic scalers: an invitro study. J Clin Diagn Res 2015;9(11):ZC56-60

6. Draenert $\mathrm{ME}$, Jakob $\mathrm{M}$, Kunzelmann $\mathrm{KH}$, Hickel R. The prevalence of tooth hyper sensitivity following periodontal therapy with special reference to root scaling. A systematic review of the literature. Am J Dent 2013;26(1):21-7.

7. Bühler J, Amato $M$, Weiger R, Walter C. A systematic review on the patient perception of periodontal treatment using air polishing devices. Int J Dent Hyg 2016;14(1):4-14.

8. Patil SS, Rakhewar PS, Limaye PS, Chaudhari NP. A comparative evaluation of plaque-removing efficacy of air polishing and rubber-cup, bristle brush with paste polish ing on oral hygiene status: a clinical study. J Int Soc Prev Community Dent 2015;5(6):45762.

9. Petersilka GJ, Bell M, Häberlein I, Mehl A Hickel R, Flemmig TF. In vitro evaluation of novel low abrasive air polishing powders. J Clin Periodontol 2003;30(1):9-13.

10. Hashino E, Kuboniwa M, Alghamdi SA Yamaguchi M, Yamamoto R, Cho $\mathrm{H}$, et al. Erythritol alters microstructure and metabolomic profiles of biofilm composed of Streptococcus gordonii and Porphyromonas gingi valis. Mol Oral Microbiol 2013;28(6):435-51. 